Gut, 1986, 27, 1403-1407

Leading article

\title{
Compliance, cost, and common sense limit cancer control in colitis
}

In this issue a Swedish group ${ }^{1}$ describe the results of a cancer surveillance programme over eight years in 71 patients with long standing extensive or substantial colitis. Their well presented study adds support to the development of such programmes which have also been reported from a number of other centres. In an elegant application of decision theory, Gage $^{2}$ has shown that a patient's five year survival is likely to be better with surveillance, despite its limitations, than with operation advised solely on the basis of extent of disease and a long history. His analysis is, however, limited to a decision late in the course of the illness and misses the point that preventive surgery to be most successful should be undertaken during the lag phase before the cancer risk begins to rise. His analysis is concerned with the early treatment of carcinoma, using dysplasia as a marker, rather than regarding colectomy for dysplasia without carcinoma as the optimal result. The ideal of colectomy at the stage of dysplasia in all patients has not so far been achieved and carcinoma tends to develop in association with dysplasia, or less commonly without, as in the Swedish series. The advantages of a surveillance programme thus have to be assessed as presumed prevention of cancer by removing a dysplastic colon in some patients and early treatment of carcinoma by colectomy in others.

The carcinoma risk in colitis is best assessed by life table analysis, though such calculations can be misunderstood and give the impression that the actual cancer risk is higher than it is. The classic actuarial analysis gives the probability of survival when every death is included as in the Registrar General's life expectancy tables. It is then possible to calculate the cumulative mortality of the original cohort in successive age periods. In calculations of cancer risk in colitis, however, many patients are withdrawn from the analysis during successive years because they have a proctocolectomy or die from various causes, particularly in older age groups. The calculation gives the risk of carcinoma among those who remain under follow up which is the clinically relevant figure because they are the patients we have to advise. To say that this risk applies to the original cohort, however, expresses the belief that those patients who had surgery or died would have been subject to a similar risk if they had lived or kept their colon. The actual risk among the original cohort is overestimated because some of the members never have the opportunity to develop carcinoma. The term life table has a connotation of mortality and it may be thought that the development of a carcinoma in colitis is invariably fatal. In fact, survival is at least $30 \%$ in the next five years in all cases, ${ }^{3}$ and greater if the tumour is treated before distant spread has occurred. ${ }^{45}$

Advice to patients is based on the duration of their disease, its extent, 
their age and general health. Carcinoma can develop within the first 10 years of the first symptoms of colitis but this is unusual. ${ }^{6}{ }^{7}$ During these years management of inflammation is the major problem and many patients with extensive colitis require colectomy for this reason so reducing the overall risk of carcinoma. Clinicians need to be alert during this period for the occasional development of dysplasia or carcinoma but regular colonoscopic screening before eight to 10 years after onset appears premature.

If inflammation is restricted to the rectum and sigmoid colon, available evidence suggests that the cancer risk is no greater than for a healthy person of the same age. ${ }^{8}$ Two cautions are needed; extensive colitis early in the illness can regress and later masquerade as distal colitis; conversely, distal colitis can extend to involve more of the colon. The former is suggested by a history of illness more severe than would be expected from distal disease, the latter can be detected by repeat investigation after any sharp relapse. At the other extreme, a patient with a barium enema showing inflammation of most or all of the colon does have a risk of carcinoma which warrants action. ${ }^{67}$ Among those patients with extensive colitis whose illness does not need surgical treatment for troublesome symptoms or chronic ill health, the annual carcinoma risk under surveillance has been estimated as about one in 80 once the total history exceeds 10 years. ${ }^{6}$ The proportion of patients who develop histological evidence of definite neoplastic potential in the colon is higher than this because additional patients accept operation for dysplasia when no carcinoma is present. These figures are of the same order as the most recent survey of 485 patients with extensive colitis, diagnosed within five years of onset in three defined geographical areas and followed for a minimum of 20 years, among whom the cumulative risk of carcinoma was $0 \cdot 6,7 \cdot 1$, and $24 \cdot 0 \%$ at 10,20 , and 30 years respectively. ${ }^{7}$ Other patients who do require regular surveillance are those in whom the rectum remains after surgical treatment, among whom the annual risk of rectal carcinoma has been estimated as about one in 200 during the second decade and one in 100 during the third decade after the onset of colitis. ${ }^{910}$

What of the patient whose barium enema appears normal or shows distal or left sided disease and yet colonoscopic biopsies show extensive disease? We do not know how to advise such a patient at present. Because barium enema has given a reasonable guide to cancer risk it seems possible that mild mucosal inflammation insufficient to cause detectable change on radiograph may not be associated with as high a carcinoma risk as the more severe disease which causes a radiographic abnormality. How about the contentious problem of colitis of intermediate extent on barium enema? In the Swedish series dysplasia was less common among this group of patients. ${ }^{1}$ It seems reasonable that there should be an excess risk among patients with disease of intermediate extent ${ }^{11}$ but in my judgment we should only attempt regular surveillance of this group when we are satisfied that we can cope with those having extensive colitis who have the greatest risk. Exceptions may be the young patient and those with a history of more than 20 years. ${ }^{11}$

Advice to patients must surely depend on their age and general health. Young patients with extensive colitis do not appear to have a greater annual risk of developing carcinoma than older patients. Colorectal 
carcinoma is so unusual in the first half of life, however, that if a young patient with colitis develops such a tumour the event rightly evokes shock and surprise. In statistical terms the relative risk, compared with the general population of the same age, may be more than a 100 -fold in the 20-40 year age group but falls (because the incidence is rising in older age groups in the general population) until it is only 10 -fold, ${ }^{6}$ or one series suggests only two-fold, ${ }^{12}$ in later years. Two other important factors in youth are long life expectancy $(90 \%$ of people aged 30 years can be expected to live more than 25 years) and social mobility, so that prolonged follow up is difficult. Can we expect a patient in the second or third decade with a 10 year history of colitis to accept regular colonoscopy for 20..30..40.. years? Can we expect continuity of medical care over a period equivalent to the average doctor's professional career? If the patient understands the position and agrees to take responsibility for seeking regular surveillance, the onus is to some extent removed from the doctor. In my opinion, the cancer risk among young patients with extensive colitis is a reasonable indication for surgical treatment now that sphincter saving surgical procedures make operation more acceptable, but if the patient understands the problem and chooses regular colonoscopy instead, surveillance is also an acceptable option.

As the years pass, the relative (but not actual) risk of carcinoma decreases, life expectancy is less, other disabilities develop, the risks of colectomy rise and restorative operations are done less frequently. All these factors tend to favour surveillance rather than prophylactic colectomy. If colitis begins after the age of 60 years, the patient will not enter the high risk group until at least the age of 70 . In this age group, many of those in good health do not wish to undergo regular colonoscopy and poor general health may preclude follow up in others.

If a surveillance programme is decided upon, how should it be organised? Apart from the special situation of the retained rectum in which rigid sigmoidoscopy is adequate, the literature speaks with one voice in saying that regular colonoscopy with multiple biopsies throughout the colon is essential. Biennial examination has maintained a low mortality from cancer in colitis but early carcinoma has been discovered with disquieting frequency. ${ }^{6}$ An interval greater than two years between colonoscopies therefore appears unwise. The Swedish group report the apparent development of a macroscopic dysplastic lesion within one year, and a carcinoma has been dicovered within nine months of a negative colonoscopic examination in another series. ${ }^{13}$ We have also seen carcinoma develop within one year of colonoscopic biopsies without dysplasia. It looks as though annual colonoscopy is desirable as long as resources are available and it is acceptable to patients. It has been estimated that for biennial examinations the staff of a district general hospital serving 270000 people would need to undertake 30 colonoscopies each year to meet the needs of its own population of patients with long standing extensive colitis. ${ }^{14}$ If the interval between examinations were halved, this would entail at least one colonoscopy a week.

Surveillance is useful only if colectomy is advised and accepted at the right time. The collaborative international study between pathologists specially interested in the topic has resulted in a consensus view on the recognition and grading of dysplasia. ${ }^{15}$ It has also shown, however, that 
interobserver error even among experts is appreciable. All are agreed that when unequivocal high grade dysplasia is found, or low grade dysplasia in association with a raised lesion, ${ }^{16}$ colectomy should be advised. The clinical significance of low grade dysplasia in flat mucosa is at present uncertain but repeat colonoscopy within a year should be the rule. Cytological changes indefinite for dysplasia often appear transient as the Swedish group observed, ${ }^{1}$ but do stimulate vigilance. In view of the inherent difficulties of distinguishing resolving inflammation from dysplasia,${ }^{17}$ and the potential interobserver error of histological interpretation, it seems wise for pathologists to ask for repeat biopsies and perhaps seek a second opinion whenever there is doubt about cytological changes.

It seems likely that cancer surveillance in colitis will become part of a gastroenterologist's regular commitment. Such a programme is difficult to sustain not only because clinical, endoscopic, and pathological work is increased, but also because patients find the examinations irksome and tend to default, which adds to the administrative work effort. There is gathering evidence that a surveillance programme will also be needed for other patients with a high risk of colorectal carcinoma, for example patients in whom multiple adenomas have been found. The needs of patients with colitis must be assessed in relation to the total resources available and the provision of a balanced service to the community. Even when cancer surveillance in colitis has been organised, the clinician must always be on the alert for the possible development of carcinoma in patients who have not been included in the programme because their risk appeared low.

St. Mark's Hospital,

J E LENNARD-JONES

London EC1. UK

\section{References}

1 Broström O, Löfberg R, Reichard $\mathrm{H}$. Cancer surveillance of patients with longstanding ulcerative colitis: a clinical, endoscopical and histological study. Gut 1986; 27 : 1408-13.

2 Gage TP. Managing the cancer risk in chronic ulcerative colitis: a decision-analytic approach. J Clin Gastroenterol 1986; 8: 50-7.

3 Gyde SN, Prior P, Thompson H, Waterhouse JA, Allan RN. Survival of patients with colorectal cancer complicating ulcerative colitis. Gut 1984; 25: 228-31.

4 Ritchie JK, Hawley PR, Lennard-Jones JE. Prognosis of carcinoma in ulcerative colitis. Gut 1981; 22: 752-5.

5 Lavery IC, Chiulli RA, Jagelman DG, Fazio VW, Weakley FL. Survival with carcinoma arising in mucosal ulcerative colitis. Ann Surg 1982; 195: 508-12.

6 Lennard-Jones JE, Morson BC, Ritchie JK, Williams CB. Cancer surveillance in ulcerative colitis: experience over 15 years. Lancet 1983; 2: 149-52.

7 Gyde SN, Stevens A, Prior P, et al. "Real" cancer risk in ulcerative colitis. [Abstract]. Gut 1986; 27: A1284-5.

8 MacDougall IPM. The cancer risk in ulcerative colitis. Lancet 1964; 2: 655-8.

9 Baker WNW, Glass RE, Ritchie JK, Aylett SO. Cancer of the rectum following colectomy and ileorectal anastomosis for ulcerative colitis. Br J Surg 1978; 65: 862-8.

10 Grundfest SF, Fazio V, Weiss RA, et al. The risk of cancer following colectomy and ileorectal anastomosis for extensive mucosal ulcerative colitis. Ann Surg 1981; 193: 9-14.

11 Greenstein AJ, Sachar DB, Smith H, et al. Cancer in universal and left-sided ulcerative colitis: factors determining risk. Gastroenterology 1979; 77: $290-4$.

12 Prior P, Gyde SN, McCartney JC, Thompson H, Waterhouse JAH, Allan RD. Cancer morbidity in ulcerative colitis. Gut 1982; 23: 490-7.

13 Allen DC, Biggart JD, Pyper PC. Large bowel mucosal dysplasia and carcinoma in ulcerative colits. J Clin Pathol 1985; 38: 30-43. 
14 Jones HW, Grogono J, Hoare AM. An audit of ulcerative colitis in a district general hospital. [Abstract]. Gut 1985; 26: A1123.

15 Riddell RH, Goldman H, Ransohoff DF, et al. Dysplasia in inflammatory bowel disease. Standardized classification with provisional clinical application. Hum Pathol 1983; 14: 931-68.

16 Blackstone MO, Riddell RH, Rogers BHG, Levin B. Dysplasia-associated lesion or mass (DALM) detected by colonoscopy in long-standing ulcerative colitis: an indication for colectomy. Gastroenterology 1981; 80: 366-74.

17 Morson BC. Precancer and cancer in inflammatory bowel disease. Pathology 1985; 17: 173-80. 